\title{
Explicit spectral gap estimates for the linearized Boltzmann and Landau operators with hard potentials
}

Céline Baranger and Clément Mouhot

\begin{abstract}
This paper deals with explicit spectral gap estimates for the linearized Boltzmann operator with hard potentials (and hard spheres). We prove that it can be reduced to the Maxwellian case, for which explicit estimates are already known. Such a method is constructive, does not rely on Weyl's Theorem and thus does not require Grad's splitting. The more physical idea of the proof is to use geometrical properties of the whole collision operator. In a second part, we use the fact that the Landau operator can be expressed as the limit of the Boltzmann operator as collisions become grazing in order to deduce explicit spectral gap estimates for the linearized Landau operator with hard potentials.
\end{abstract}

\section{Introduction}

This paper is devoted to the study of the spectral properties of the linearized Boltzmann and Landau collision operators with hard potentials. In this work we shall obtain new quantitative estimates on the spectral gap of these operators. Before we explain our methods and results in more details, let us introduce the problem in a precise way. The Boltzmann equation describes the behavior of a dilute gas when the only interactions taken into account are binary elastic collisions. It reads in $\mathbb{R}^{N}(N \geq 2)$

$$
\frac{\partial f}{\partial t}+v \cdot \nabla_{x} f=Q^{\mathcal{B} o}(f, f),
$$

where $f(t, x, v)$ stands for the time-dependent distribution function of density of particles in the phase space.

2000 Mathematics Subject Classification: Primary 76P05; Second.: 82B40, 82C40, 82D05. Keywords: spectral gap, linearized Boltzmann operator, Landau linearized operator, geometrical properties, explicit, grazing collision limit, hard potentials. 
The $N$-dimensional Boltzmann collision operator $Q$ is a quadratic operator, which is local in $(t, x)$. The time and position are only parameters and therefore shall not be written in the sequel: the estimates proven in this paper are all local in $(t, x)$. Thus it acts on $f(v)$ by

$$
Q^{\mathcal{B} o}(f, f)(v)=\int_{v_{*} \in \mathbb{R}^{N}} \int_{\sigma \in \mathbb{S}^{N-1}} B\left(\cos \theta,\left|v-v_{*}\right|\right)\left[f_{*}^{\prime} f^{\prime}-f_{*} f\right] d \sigma d v_{*}
$$

where we have used the shorthands $f=f(v), f_{*}=f\left(v_{*}\right), f^{\prime}=f\left(v^{\prime}\right)$, $f_{*}^{\prime}=f\left(v_{*}^{\prime}\right)$. The velocities are given by

$$
v^{\prime}=\frac{v+v_{*}}{2}+\frac{\left|v-v_{*}\right|}{2} \sigma, \quad v_{*}^{\prime}=\frac{v+v^{*}}{2}-\frac{\left|v-v_{*}\right|}{2} \sigma .
$$

The collision kernel $B$ is a non-negative function which only depends on $\left|v-v_{*}\right|$ and $\cos \theta=k \cdot \sigma$ where $k=\left(v-v_{*}\right) /\left|v-v_{*}\right|$.

Consider the collision operator obtained by the linearization process around the Maxwellian global equilibrium state denoted by $M$

$$
L^{\mathcal{B} o} h(v)=\int_{\mathbb{R}^{N}} \int_{\mathbb{S}^{N-1}} B\left(\cos \theta,\left|v-v_{*}\right|\right) M\left(v_{*}\right)\left[h_{*}^{\prime}+h^{\prime}-h_{*}-h\right] d \sigma d v_{*},
$$

where $f=M(1+h)$ and $M(v)=e^{-|v|^{2}}$. Notice that for the associated linearized equation, the entropy is the $L^{2}(M)$ norm of $h$ and thus by differentiating, the entropy production is

$$
\begin{aligned}
& <h, L^{\mathcal{B} o} h>_{L^{2}(M)}= \\
& \quad=-\frac{1}{4} \int_{\mathbb{R}^{N}} \int_{\mathbb{R}^{N}} \int_{\mathbb{S}^{N-1}} B\left(\cos \theta,\left|v-v_{*}\right|\right)\left[h_{*}^{\prime}+h^{\prime}-h_{*}-h\right]^{2} M M_{*} d \sigma d v_{*} d v .
\end{aligned}
$$

This quantity is non-positive. At the level of the linearized equation, this corresponds to the first part of Boltzmann's $H$-theorem, and it implies that the spectrum of $L^{\mathcal{B} o}$ in $L^{2}(M)$ is non-positive. Let us denote

$$
D^{\mathcal{B} o}(h)=-<h, L^{\mathcal{B o}} h>_{L^{2}(M)} .
$$

We shall call this quantity "(linearized) entropy production functional" by analogy with the nonlinear case.

In the case of long-distance interaction, the collisions occur mostly for very small deviation angle $\theta$. In the case of the Coulomb potential, for which the Boltzmann collision operator is meaningless (see [18, Annex I, Appendix A]), one has to replace it by the Landau collision operator

$$
Q^{\mathcal{L} a}(f, f)(v)=\nabla_{v} \cdot\left(\int_{v_{*} \in \mathbb{R}^{N}} \mathbf{a}\left(v-v_{*}\right)\left[f_{*}(\nabla f)-f(\nabla f)_{*}\right] d v_{*}\right),
$$

with $\mathbf{a}(z)=|z|^{2} \Phi(z) \Pi_{z^{\perp}}$, where $\Pi_{z^{\perp}}$ is the orthogonal projection onto $z^{\perp}$, i.e.,

$$
\left(\Pi_{z^{\perp}}\right)_{i, j}=\delta_{i, j}-\frac{z_{i} z_{j}}{|z|^{2}}
$$


This operator is used for instance in models of plasma in the case of a Coulomb potential, i.e., a gas of (partially or totally) ionized particles (for more details see [16] and the references therein). Applying the same linearization process than for the Boltzmann operator (around the same global equilibrium $M$ ), we define the linearized Landau operator

$$
L^{\mathcal{L} a} h(v)=M(v)^{-1} \nabla_{v} \cdot\left(\int_{v_{*} \in \mathbb{R}^{N}} \mathbf{a}\left(v-v_{*}\right)\left[(\nabla h)-(\nabla h)_{*}\right] M M_{*} d v_{*}\right),
$$

and the (linearized) Landau entropy production functional

$$
\begin{aligned}
D^{\mathcal{L} a}(h) & =-<h, L^{\mathcal{L} a} h>_{L^{2}(M)} \\
& =\frac{1}{2} \int_{\mathbb{R}^{N}} \int_{\mathbb{R}^{N}} \Phi\left(v-v_{*}\right)\left|v-v_{*}\right|^{2}\left\|\Pi_{\left(v-v_{*}\right)^{\perp}}\left[(\nabla h)-(\nabla h)_{*}\right]\right\|^{2} M M_{*} d v_{*} d v
\end{aligned}
$$

which is also non-positive. It implies that the spectrum of $L^{\mathcal{L} a}$ in $L^{2}(M)$ is non-positive.

Let us now write down our assumptions for the collision kernel $B$ :

- $B$ is a tensorial product

$$
B=b(\cos \theta) \Phi\left(\left|v-v_{*}\right|\right),
$$

where $\Phi$ and $b$ are non-negative functions (this is the case for instance for collision kernels deriving from interaction potentials behaving like inverse-power laws).

- The kinetic part $\Phi$ is bounded from below at infinity, i.e.,

$$
\exists R \geq 0, c_{\Phi}>0 \quad \mid \forall r \geq R, \Phi(r) \geq c_{\Phi} .
$$

This assumption holds for hard potentials (and hard spheres).

- The angular part $b$ satisfies

$$
c_{b}=\inf _{\sigma_{1}, \sigma_{2} \in \mathbb{S}^{N-1}} \int_{\sigma_{3} \in \mathbb{S}^{N-1}} \min \left\{b\left(\sigma_{1} \cdot \sigma_{3}\right), b\left(\sigma_{2} \cdot \sigma_{3}\right)\right\} d \sigma_{3}>0 .
$$

This covers all the physical cases.

Remarks: 1 . Notice that there is no $b$ left in $Q^{\mathcal{L} a}$ and $L^{\mathcal{L} a}$ but the function $\Phi$ is definitely the same in both Landau and Boltzmann operators. Therefore the assumptions on the Landau operator reduce to (1.2). Thus we deal with the so-called "hard potentials" case for the Landau operator, which excludes the Coulomb potential. 
2. The assumption that $B$ is a tensorial product is made for a sake of simplicity. Indeed, one could easily adapt the proofs in section 2 to relax this assumption. The price to pay would be a more technical condition on the collision kernel $B$.

The spectral properties of the linearized Boltzmann and Landau operators have been extensively studied. In particular, there are of crucial interest for perturbative approach issues. For instance, the convergence to equilibrium has been studied in this context, as well as the hydrodynamical limit (see [12]).

On the one hand, for hard potentials, the existence of a spectral gap as soon as the kinetic part of the collision kernel is bounded from below at infinity is a classical result, which can be traced back unto Grad himself. The only method was up to now to work under the assumption of Grad's angular cutoff, and to apply Weyl's Theorem to $L^{\mathcal{B}}$, written as a compact perturbation of a multiplication operator (a very clear presentation of this proof can be found in [5]). The picture of the spectrum obtained for the operator (under Grad's cutoff assumption) is described by figures 1 and 2 (see [4]).

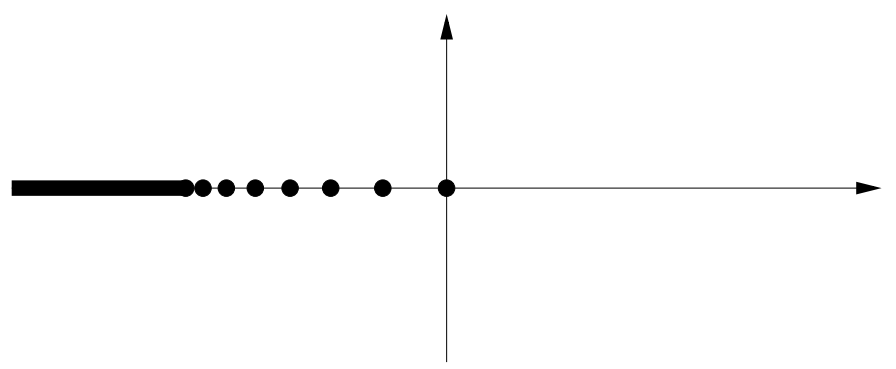

FiguRE 1: Spectrum of the collision operator for strictly hard potential with angular cutoff.

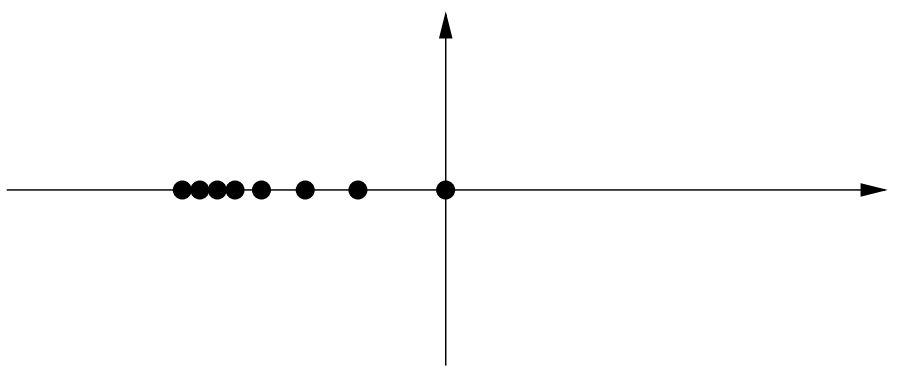

FiguRE 2: Spectrum of the collision operator for Maxwell's molecules with angular cutoff.

A similar method has been applied to the linearized Landau operator with hard potential in [7]. 
On the other hand, for the particular case of Maxwellian molecules (for $L^{\mathcal{B}}$ ), a complete and explicit diagonalisation has been obtained first by symmetry arguments in [19], and then by Fourier methods in [1]. The spectral gap for the "over-Maxwellian" collision kernel of the linearized Landau operator (i.e., collision kernels which are bounded from below by one for Maxwellian molecules) can be derived from results in [11], by a linearization process. Notice also that in the case of the so-called Kac's equation, an explicit entropy production estimate, based on a cancellation method, was given in [10]; this method can be linearized in order to give explicit spectral gap estimates for "over-quadratic" linearized Kac's operator (for which the physical meaning is not clear!). Nevertheless we did not manage to adapt this strategy to the Boltzmann operator with hard potentials. Notice however that Wennberg [20] gave an extension of the very first entropy estimates of Desvillettes [8] to allow for hard and soft potentials. His idea has some similarities with ours: to avoid the region in $\mathbb{R}^{N} \times \mathbb{R}^{N}$ where $\Phi\left(\left|v-v_{*}\right|\right)$ is small.

A specific study of the spectral properties of the linearized operator was made for non-cutoff hard potentials in [15]. Nevertheless this article was critically reviewed some years later in [14]. Also another specific study for "radial cutoff potentials" was done in [6].

Finally notice that it is proved in [2] that the linearized Boltzmann operator for soft potential with angular cutoff has no spectral gap. The resulting spectrum is described in figure 3 (see [4]).

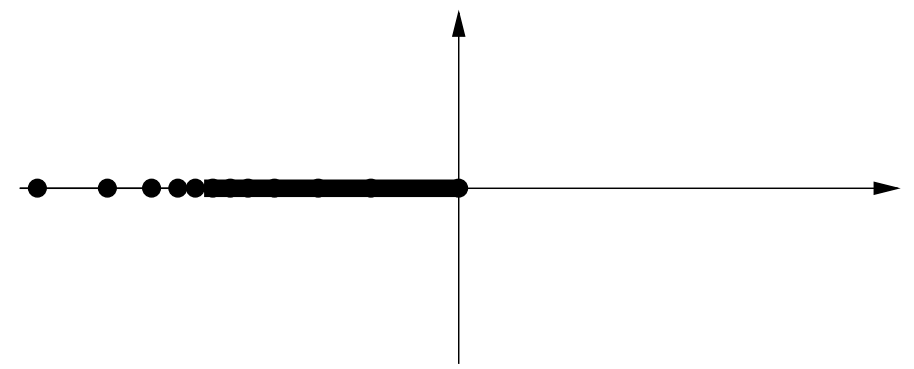

FiguRE 3: Spectrum of the collision operator for soft potentials with angular cutoff.

But if one allows a loss on the algebraic weight of the norm, it was proved in [13] a "degenerated spectral gap" result of the form

$$
\left\|L^{\mathcal{B} o} h\right\|_{L^{2}(M)} \geq C\|h\|_{L_{\gamma}^{2}(M)} \forall h \perp\left\{1 ; v ;|v|^{2}\right\}
$$

where $\gamma<0$ is the power of the kernel $\Phi$. It is based on inequalities proven in [2] and Weyl's Theorem. 
However, the perturbative method has drawbacks, all coming from the fact that it does not rely on a physical argument. First it is not explicit, that is the width of the spectral gap is not known, which is problematic when one wants to obtain quantitative estimates of convergence to equilibrium. Secondly it gives no information about how this spectral gap is sensitive to the perturbation of the collision kernel. Finally approaches based on Weyl's Theorem rely strongly on Grad's cutoff assumption via "Grad's splitting", which means to deal separately with the gain and the loss part of the collision operator.

Our method is geometrical and based on a physical argument. It gives explicit estimates and deals with the whole operator, with or without angular cutoff. Up to our knowledge, as far as spectral gaps are considered, it covers all the results of the above-mentioned articles dealing with hard potentials, with or without angular cutoff.

We think likely that this geometrical method could also be adapted to give explicit versions of "degenerated spectral gap" results in the case of soft potentials, even if up to now we did not manage to do it.

We now state our main theorems:

Theorem 1.1. (The linearized Boltzmann operator) Under the assumptions (1.1), (1.2), (1.3), the Boltzmann entropy production functional $D^{\mathcal{B} o}$ with $B=\Phi b$ satisfies, for all $h \in L^{2}(M)$

$$
D^{\mathcal{B} o}(h) \geq C_{\Phi, b}^{\mathcal{B} o} D_{0}^{\mathcal{B} o}(h)
$$

where $D_{0}^{\mathcal{B} o}(h)$ stands for the entropy production functional with $B_{0} \equiv 1$ and

$$
C_{\Phi, b}^{\mathcal{B} o}=\left(\frac{c_{\Phi} c_{b} e^{-4 R^{2}}}{32\left|\mathbb{S}^{N-1}\right|}\right)
$$

with $R, c_{\Phi}, c_{b}$ being defined in (1.2), (1.3).

As a consequence we deduce quantitative estimates on the spectral gap of the linearized Boltzmann operator, namely for all $h \in L^{2}(M)$ orthogonal in $L^{2}(M)$ to $1, v$ and $|v|^{2}$, we have

$$
D^{\mathcal{B} o}(h) \geq C_{\Phi . b}^{\mathcal{B} o}\left|\lambda_{0}^{\mathcal{B} o}\right|\|h\|_{L^{2}(M)}^{2} .
$$

Here $\lambda_{0}^{\mathcal{B} o}$ is the first non-zero eigenvalue of the linearized Boltzmann operator with $B_{0} \equiv 1$ (that is, for Maxwellian molecules with no angular dependence, sometimes called pseudo-Maxwellian molecules) which equals in dimension 3 (see [1])

$$
\lambda_{0}^{\mathcal{B} o}=-\pi^{5 / 2} \int_{0}^{\pi} \sin ^{3} \theta d \theta=-\frac{4}{3} \pi^{5 / 2} .
$$


Remark: As an application of this theorem, let us give explicit formulas for the spectral gap $S_{\gamma}^{\mathcal{B} o}$ of the linearized Boltzmann operator with $b \geq 1$ and $\Phi(z)=|z|^{\gamma}, \gamma>0$, in dimension 3. Then $c_{b} \geq\left|\mathbb{S}^{2}\right|$ and for any given $R$ we can take $c_{\Phi}=R^{\gamma}$. Thus we get

$$
S_{\gamma}^{\mathcal{B} o} \geq\left(\frac{R^{\gamma} e^{-4 R^{2}}}{32}\right) \frac{4}{3} \pi^{5 / 2}
$$

for any $R>0$. An easy computation leads to the lower bound

$$
S_{\gamma}^{\mathcal{B} o} \geq \frac{\pi^{5 / 2}(\gamma / 8)^{\gamma / 2} e^{-\gamma / 2}}{24}
$$

by optimizing the free parameter $R$.

Theorem 1.2. (The Landau linearized operator) Under assumptions (1.2), the Landau entropy production functional $D^{\mathcal{L} a}$ with collision kernel $\Phi$ satisfies, for all $h \in L^{2}(M)$

$$
D^{\mathcal{L} a}(h) \geq C_{\Phi}^{\mathcal{L} a} D_{0}^{\mathcal{L} a}(h)
$$

where $D_{0}^{\mathcal{L} a}(h)$ stands for the Landau entropy production functional with $\Phi_{0} \equiv 1$ and

$$
C_{\Phi}^{\mathcal{L} a}=\left(\frac{c_{\Phi} \beta_{R}}{8 \alpha_{N}}\right)
$$

with

$$
\alpha_{N}=\int_{\mathbb{R}^{N-1}} e^{-|V|^{2}} d V, \quad \beta_{R}=\int_{\left\{V \in \mathbb{R}^{N-1}|| V \mid \geq 2 R\right\}} e^{-|V|^{2}} d V .
$$

As a consequence we deduce quantitaves estimates on the spectral gap of the linearized Landau operator, namely for all $h \in L^{2}(M)$ orthogonal in $L^{2}(M)$ to $1, v$ and $|v|^{2}$, we have

$$
D^{\mathcal{L} a}(h) \geq C_{\Phi}^{\mathcal{L} a}\left|\lambda_{0}^{\mathcal{L} a}\right|\|h\|_{L^{2}(M)}^{2} .
$$

Here $\lambda_{0}^{\mathcal{L} a}$ is the first non-zero eigenvalue of the linearized Landau operator with $\Phi_{0} \equiv 1$ (that is, for Maxwellian molecules).

Moreover in dimension 3, by grazing collisions limit, we can estimate $\lambda_{0}^{\mathcal{L} a}$ thanks to the explicit formula on the spectral gap of the linearized Boltzmann operator for Maxwellian molecules

$$
\left|\lambda_{0}^{\mathcal{L} a}\right| \geq 2 \pi
$$


Remarks: 1. As for the linearized Boltzmann operator, we can deduce from this theorem an explicit formula for a lower bound on the spectral gap $S_{\gamma}^{\mathcal{L a}}$ for the linearized Landau operator with hard potentials $\Phi(z)=|z|^{\gamma}, \gamma>0$, in dimension 3. We get

$$
S_{\gamma}^{\mathcal{L} a} \geq\left(\frac{R^{\gamma} e^{-4 R^{2}}}{8}\right) 2 \pi^{5 / 2}
$$

for any $R>0$. An easy computation leads to the lower bound

$$
S_{\gamma}^{\mathcal{L} a} \geq \frac{\pi^{5 / 2}(\gamma / 8)^{\gamma / 2} e^{-\gamma / 2}}{4}
$$

by optimizing the free parameter $R$.

2. The modulus of the first non-zero eigenvalue of the linearized Landau operator for Maxwellian molecules is estimated here by grazing collisions limit. Other methods would have been the linearization of entropy estimates in [11], or to use the decomposition (established in [17]) of the Landau operator for Maxwellian molecules into a Fokker-Planck part (for which spectral gap is already known) and a spherical diffusion process, which can only increases the spectral gap; and then to linearize the estimate thus obtained.

3. More generally, it is likely that an explicit spectral gap for the linearized Landau operator with hard potentials could be directly computed by existing methods even if up to our knowledge this is the first explicit formula. But Theorem 1.2 is stronger: it says that the property proved on the Boltzmann operator with hard potentials, namely "cancellations for small relative velocities can be neglected as far as entropy production is concerned", remains true for the linearized Landau operator with hard potentials.

The idea of the proof is to reduce the case of hard potentials (in the generalized sense (1.2)) to the Maxwellian case. The difficulty is to deal with the cancellations of the kinetic collision kernel $\Phi$ on the diagonal $v=v_{*}$.

The starting point is the following inequality which is a corollary of $[3$, Theorem 2.4]

$$
\begin{aligned}
\int_{\mathbb{R}^{N}} \int_{\mathbb{R}^{N}}|\xi(x)-\xi(y)|^{2}|x-y|^{\gamma} M(x) M(y) d x d y & \\
& \geq K_{\gamma} \int_{\mathbb{R}^{N}} \int_{\mathbb{R}^{N}}|\xi(x)-\xi(y)|^{2} M(x) M(y) d x d y
\end{aligned}
$$

for $\gamma \geq 0, \xi$ some function, and

$$
K_{\gamma}=\frac{1}{4 \int_{\mathbb{R}^{N}} M} \inf _{x, y \in \mathbb{R}^{N}} \int_{\mathbb{R}^{N}} \min \left\{|x-z|^{\gamma},|z-y|^{\gamma}\right\} M(z) d z .
$$


It was first suggested by Villani [16, Chap. 5, section 1.4], in the context of the study of entropy-entropy dissipation inequalities for the Landau equation with hard potentials, that this inequality could allow to prove that hard potentials reduce to the Maxwellian case as far as convergence to equilibrium is concerned.

The proof of (1.9) relies strongly on the existence of a "triangular inequality" for some function $F(x, y)$ integrated: in (1.9), the function $F$ is simply $|\xi(x)-\xi(y)|^{2}$ which satisfies

$$
F(x, y) \leq 2 F(x, z)+2 F(z, y) .
$$

The main difficulty is hence to obtain such a "triangular inequality" adapted to our case for the linearized Boltzmann operator. It will be discussed in details in section 2 together with the proof of Theorem 1.1. Section 3 will be devoted to the linearized Landau operator: using results of section 2, we will prove Theorem 1.2 thanks to a grazing collision limit.

\section{The linearized Boltzmann operator}

In this section, we present the proof of inequality (1.4) in Theorem 1.1. In order to "avoid" the diagonal $v \sim v_{*}$ where $\Phi$ is not uniformly bounded from below, we use the following argument: perfoming a collision with small relative velocity (i.e., for a small $\left.\left|v-v_{*}\right|\right)$ is the same than perfoming two collisions with great relative velocity, provided that the pre- and post-collisionnal velocities are the same. One could summarize the situation in this way: when a collision with small relative velocity occurs, at the same time, two collisions with great relative velocity occur, which give the same pre- and post-collisionnal velocities, and which produce at least the same amount of entropy.

Before proving (1.4), let us begin with a preliminary lemma dealing with the angular part of the collision kernel. This lemma is based on the same geometrical idea as the one we shall use for the treatment of the cancellations of $\Phi$ : the introduction of some well-chosen intermediate collision. This first step is made for the sake of simplicity: we show that in the sequel of this section one can set $b \equiv 1$ without restriction. It makes the proof clearer, and simplifies somehow the constants.

Let us denote from now on

$$
k\left(v, v_{*}, v^{\prime}, v_{*}^{\prime}\right)=\left[h(v)+h\left(v_{*}\right)-h\left(v^{\prime}\right)-h\left(v_{*}^{\prime}\right)\right]^{2} .
$$


Lemma 2.1. (Homogeneization of the angular collision kernel $\boldsymbol{b}$ ) Under the assumptions (1.1), (1.2), (1.3), for all $h \in L^{2}(M)$,

$$
D^{\mathcal{B} o}(h) \geq \frac{c_{b}}{4\left|\mathbb{S}^{N-1}\right|} D_{1}^{\mathcal{B} o}(h)
$$

where $D_{1}^{\mathcal{B} o}$ denotes the entropy production functional with $B=\Phi\left(\left|v-v_{*}\right|\right)$ instead of $B=\Phi\left(\left|v-v_{*}\right|\right) b(\theta)$.

Remark: This lemma allows to bound from below the entropy production functional by one with an "uniform angular collision kernel", i.e., a constant $c_{b}$, even when $b$ is not bounded from below by a positive number uniformly on the sphere. Notice for instance that the condition $c_{b}>0$ is satisfied for $b$ having only finite number of 0 .

Proof of Lemma 2.1. First, we write down an appropriate representation of the operator. The functional $D^{\mathcal{B} o}$ reads in " $\sigma$-representation"

$D^{\mathcal{B} o}(h)=\frac{1}{4} \int_{\mathbb{R}^{N}} \int_{\mathbb{R}^{N}} \int_{\mathbb{S}^{N-1}} \Phi\left(\left|v-v_{*}\right|\right) b\left(\frac{v-v_{*}}{\left|v-v_{*}\right|} \cdot \sigma_{1}\right) M M_{*} k\left(v, v_{*}, v^{\prime}, v_{*}^{\prime}\right) d \sigma_{1} d v_{*} d v$.

(for the classical representations of the Boltzmann operator we refer to [16]). Then keeping $\sigma_{1}$ fixed we do the change of variable $\left(v, v_{*}\right) \rightarrow\left(\frac{v+v_{*}}{2}, \frac{v-v_{*}}{2}\right)$, whose jacobian is $(-1 / 2)^{N}$. Let us denote $\Omega=\frac{v+v_{*}}{2}$ and $\Omega^{\prime}=\frac{v-v_{*}}{2}$. We obtain

$$
\begin{aligned}
D^{\mathcal{B} o}(h)= & \frac{2^{N}}{4} \int_{\Omega \in \mathbb{R}^{N}} \int_{\Omega^{\prime} \in \mathbb{R}^{N}} \int_{\mathbb{S}^{N-1}} \Phi\left(2\left|\Omega^{\prime}\right|\right) b\left(\frac{\Omega^{\prime}}{\left|\Omega^{\prime}\right|} \cdot \sigma_{1}\right) \\
& k\left(\Omega+\Omega^{\prime}, \Omega-\Omega^{\prime}, \Omega+\left|\Omega^{\prime}\right| \sigma_{1}, \Omega-\left|\Omega^{\prime}\right| \sigma_{1}\right) e^{-2|\Omega|^{2}-2\left|\Omega^{\prime}\right|^{2}} d \sigma_{1} d \Omega d \Omega^{\prime}
\end{aligned}
$$

(recall that $|\Omega|^{2}+\left|\Omega^{\prime}\right|^{2}=\frac{|v|^{2}+\left|v_{*}\right|^{2}}{2}$ ).

We now write $\Omega^{\prime}$ in spherical coordinates $\Omega^{\prime}=r \sigma_{2}$, the other variables being kept fixed, and use Fubini's Theorem

$$
\begin{gathered}
D^{\mathcal{B} o}(h)=\frac{2^{N}}{4} \int_{\Omega \in \mathbb{R}^{N}} \int_{r \in \mathbb{R}_{+}} r^{N-1} \Phi(2 r) e^{-2|\Omega|^{2}-2 r^{2}} \int_{\sigma_{1} \in \mathbb{S}^{N-1}} \int_{\sigma_{2} \in \mathbb{S}^{N-1}} b\left(\sigma_{1} \cdot \sigma_{2}\right) \\
k\left(\Omega+r \sigma_{2}, \Omega-r \sigma_{2}, \Omega+r \sigma_{1}, \Omega-r \sigma_{1}\right) d \sigma_{1} d \sigma_{2} d r d \Omega .
\end{gathered}
$$

Now we apply a geometrical idea that we shall also use below in the treatment of cancellations of $\Phi$ : namely we add a third artificial variable. Let us thus introduce two collisions points $u$ and $u_{*}$ on the sphere of center $\Omega$ and radius $r$ (see figure 4) and replace the collision " $\left(v, v_{*}\right)$ gives $\left(v^{\prime}, v_{*}^{\prime}\right)$ " by the two collisions " $\left(v, v_{*}\right)$ gives $\left(u, u_{*}\right)$ " and " $\left(u, u_{*}\right)$ gives $\left(v^{\prime}, v_{*}^{\prime}\right)$ ". 


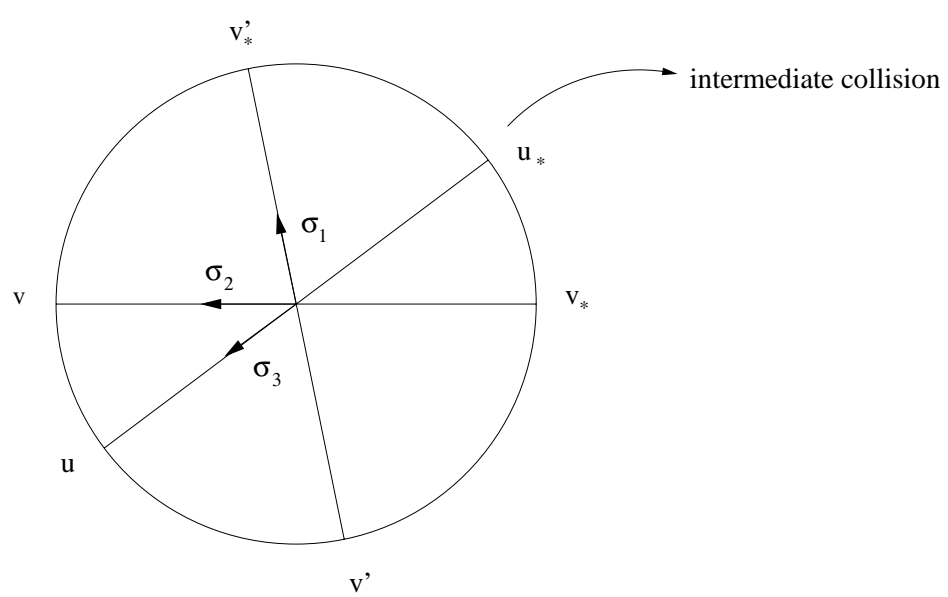

FigURE 4: Introduction of an intermediate collision.

Then, we shall use the following "triangular" inequality on the collision points:

$$
\begin{aligned}
{\left[\left(h(v)+h\left(v_{*}\right)\right)-\left(h\left(v^{\prime}\right)+h\left(v_{*}^{\prime}\right)\right)\right]^{2} \leq } & 2\left[\left(h(v)+h\left(v_{*}\right)\right)-\left(h(u)+h\left(u_{*}\right)\right)\right]^{2} \\
& +2\left[\left(h(u)+h\left(u_{*}\right)\right)-\left(h\left(v^{\prime}\right)+h\left(v_{*}^{\prime}\right)\right)\right]^{2} .
\end{aligned}
$$

So let us add a third "blind" variable $\sigma_{3}$ on the sphere

$$
\begin{aligned}
D^{\mathcal{B} o}(h)= & \frac{2^{N}}{4\left|\mathbb{S}^{N-1}\right|} \int_{\Omega \in \mathbb{R}^{N}} \int_{r \in \mathbb{R}_{+}} r^{N-1} \Phi(2 r) e^{-2|\Omega|^{2}-2 r^{2}} \int_{\sigma_{1} \in \mathbb{S}^{N-1}} \int_{\sigma_{2} \in \mathbb{S}^{N-1}} \int_{\sigma_{3} \in \mathbb{S}^{N-1}} \\
& b\left(\sigma_{1} \cdot \sigma_{2}\right) k\left(\Omega+r \sigma_{2}, \Omega-r \sigma_{2}, \Omega+r \sigma_{1}, \Omega-r \sigma_{1}\right) d \sigma_{1} d \sigma_{2} d \sigma_{3} d r d \Omega .
\end{aligned}
$$

As variables $\sigma_{1}, \sigma_{2}$ and $\sigma_{3}$ are equivalent, one can change the "blind" variable into either $\sigma_{1}$ or $\sigma_{2}$ and compute the mean to get

$$
\begin{aligned}
D^{\mathcal{B} o}(h)= & \frac{2^{N}}{4\left|\mathbb{S}^{N-1}\right|} \int_{\Omega \in \mathbb{R}^{N}} \int_{r \in \mathbb{R}_{+}} r^{N-1} \Phi(2 r) e^{-2|\Omega|^{2}-2 r^{2}} \int_{\sigma_{1} \in \mathbb{S}^{N-1}} \int_{\sigma_{2} \in \mathbb{S}^{N-1}} \int_{\sigma_{3} \in \mathbb{S}^{N-1}} \\
& \frac{1}{2}\left[b\left(\sigma_{1} \cdot \sigma_{3}\right) k\left(\Omega+r \sigma_{3}, \Omega-r \sigma_{3}, \Omega+r \sigma_{1}, \Omega-r \sigma_{1}\right)\right. \\
& \left.+b\left(\sigma_{2} \cdot \sigma_{3}\right) k\left(\Omega+r \sigma_{2}, \Omega-r \sigma_{2}, \Omega+r \sigma_{3}, \Omega-r \sigma_{3}\right)\right] d \sigma_{1} d \sigma_{2} d \sigma_{3} d r d \Omega,
\end{aligned}
$$

which yields

$$
\begin{aligned}
D^{\mathcal{B} o}(h) & \geq \frac{2^{N}}{4 \mid \mathbb{S}^{N-1 \mid}} \int_{\Omega \in \mathbb{R}^{N}} \int_{r \in \mathbb{R}_{+}} \int_{\sigma_{1} \in \mathbb{S}^{N-1}} \int_{\sigma_{2} \in \mathbb{S}^{N-1}} \int_{\sigma_{3} \in \mathbb{S}^{N-1}} \Phi(2 r) \\
& \frac{1}{2} \min \left\{b\left(\sigma_{1} \cdot \sigma_{3}\right), b\left(\sigma_{2} \cdot \sigma_{3}\right)\right\}\left[k\left(\Omega+r \sigma_{3}, \Omega-r \sigma_{3}, \Omega+r \sigma_{1}, \Omega-r \sigma_{1}\right)\right. \\
& \left.+k\left(\Omega+r \sigma_{2}, \Omega-r \sigma_{2}, \Omega+r \sigma_{3}, \Omega-r \sigma_{3}\right)\right] e^{-2|\Omega|^{2}-2 r^{2}} d \sigma_{1} d \sigma_{2} d \sigma_{3} d r d \Omega .
\end{aligned}
$$


The triangular inequality needed on $k$ is

$$
\begin{aligned}
k\left(\Omega+r \sigma_{2}, \Omega-\right. & \left.r \sigma_{2}, \Omega+r \sigma_{1}, \Omega-r \sigma_{1}\right) \\
\leq & 2 k\left(\Omega+r \sigma_{3}, \Omega-r \sigma_{3}, \Omega+r \sigma_{1}, \Omega-r \sigma_{1}\right) \\
& +2 k\left(\Omega+r \sigma_{2}, \Omega-r \sigma_{2}, \Omega+r \sigma_{3}, \Omega-r \sigma_{3}\right)
\end{aligned}
$$

and follows from (2.2). Thus if one sets

$$
c_{b}=\inf _{\sigma_{1}, \sigma_{2} \in \mathbb{S}^{N-1}} \int_{\sigma_{3} \in \mathbb{S}^{N-1}} \min \left\{b\left(\sigma_{1} \cdot \sigma_{3}\right), b\left(\sigma_{2} \cdot \sigma_{3}\right)\right\} d \sigma_{3},
$$

one obtains (going back to the classical representation)

$$
\begin{aligned}
D^{\mathcal{B} o}(h) & \geq \frac{c_{b}}{4\left|\mathbb{S}^{N-1}\right|} \frac{1}{4} \int_{\mathbb{S}^{N-1}} \int_{\mathbb{R}^{N}} \int_{\mathbb{R}^{N}} \Phi\left(\left|v-v_{*}\right|\right) M M_{*} k\left(v, v_{*}, v^{\prime}, v_{*}^{\prime}\right) d \sigma d v_{*} d v \\
& \geq \frac{c_{b}}{4\left|\mathbb{S}^{N-1}\right|} D_{1}^{\mathcal{B}_{0}}(h)
\end{aligned}
$$

which concludes the proof.

Lemma 2.2. (Treatment of the cancellations of $\Phi$ ) Under the assumption (1.2) on $\Phi$, for all $h \in L^{2}(M)$

$$
D_{1}^{\mathcal{B} o}(h) \geq\left(\frac{c_{\Phi} e^{-4 R^{2}}}{8}\right) D_{0}^{\mathcal{B} o}(h)
$$

where $D_{1}^{\mathcal{B} o}$ is the entropy production functional with $B=\Phi\left(\left|v-v_{*}\right|\right)$ and $D_{0}^{\mathcal{B} o}$ is the entropy production functional with $B=1$.

Proof of Lemma 2.2. We assume here that $b \equiv 1$. Lemma 2.1 indeed shows that this is no restriction modulo a factor $c_{b} /\left(4\left|\mathbb{S}^{N-1}\right|\right)$. Let us consider the so-called " $\omega$-representation" (see [16] again): the vector $\sigma$ integrated on the sphere becomes $\omega=\frac{v^{\prime}-v}{\left|v^{\prime}-v\right|}$ and the change of variable changes the angular kernel into

$$
\tilde{b}(\theta)=2^{N-1} \sin ^{N-2}\left(\frac{\theta}{2}\right)
$$

where $\cos \theta=2(k \cdot \omega)^{2}-1$ with $k=\left(v-v_{*}\right) /\left|v-v_{*}\right|$.

The operator $D_{1}^{\mathcal{B} o}(h)$ thus becomes

$$
D_{1}^{\mathcal{B} o}(h)=\frac{1}{4} \int_{\mathbb{R}^{N}} \int_{\mathbb{R}^{N}} \int_{\mathbb{S}^{N-1}} \Phi\left(\left|v-v_{*}\right|\right) \tilde{b}(\theta) M M_{*} k\left(v, v_{*}, v^{\prime}, v_{*}^{\prime}\right) d \omega d v d v_{*}
$$


where the velocities $v^{\prime}, v_{*}^{\prime}$ are given by

$$
v^{\prime}=v-\left(v-v_{*}, \omega\right) \omega, \quad v_{*}^{\prime}=v_{*}+\left(v-v_{*}, \omega\right) \omega .
$$

Then keeping $\omega$ fixed we do the following change of variable

$$
v=r_{1} \omega+V_{1}, \quad v_{*}=r_{2} \omega+V_{2}
$$

with $V_{1}, V_{2} \in \omega^{\perp}$. The Jacobian of the change of variable is 1 since the decompositions are orthogonal. Finally we obtain the following representation

$$
\begin{aligned}
D_{1}^{\mathcal{B} o}(h)= & \frac{1}{4} \int_{\mathbb{S}^{N-1}} \int_{V_{1} \in \omega^{\perp}} \int_{V_{2} \in \omega^{\perp}} e^{-\left|V_{1}\right|^{2}-\left|V_{2}\right|^{2}} \\
& \int_{r_{1} \in \mathbb{R}} \int_{r_{2} \in \mathbb{R}} e^{-r_{1}^{2}-r_{2}^{2}} \Phi\left(\sqrt{\left|r_{2}-r_{1}\right|^{2}+\left|V_{2}-V_{1}\right|^{2}}\right) \\
& \tilde{b}(\theta) k\left(r_{1} \omega+V_{1}, r_{2} \omega+V_{2}, r_{2} \omega+V_{1}, r_{1} \omega+V_{2}\right) d r_{1} d r_{2} d V_{2} d V_{1} d \omega .
\end{aligned}
$$

Assume that $\Phi$ is non-decreasing. This is no restriction since $\Phi \geq \tilde{\Phi}$, with

$$
\tilde{\Phi}(r)=\inf _{r^{\prime} \geq r} \Phi\left(r^{\prime}\right)
$$

and $\tilde{\Phi}$ satisfies assumption (1.2) with the same constant as $\Phi$. This monotonicity yields

$$
\begin{aligned}
D_{1}^{\mathcal{B} o}(h) \geq & \frac{1}{4} \int_{\mathbb{S}^{N-1}} \int_{V_{1} \in \omega^{\perp}} \int_{V_{2} \in \omega^{\perp}} e^{-\left|V_{1}\right|^{2}-\left|V_{2}\right|^{2}} \int_{r_{1} \in \mathbb{R}} \int_{r_{2} \in \mathbb{R}} e^{-r_{1}^{2}-r_{2}^{2}} \Phi\left(\left|r_{2}-r_{1}\right|\right) \\
& \tilde{b}(\theta) k\left(r_{1} \omega+V_{1}, r_{2} \omega+V_{2}, r_{2} \omega+V_{1}, r_{1} \omega+V_{2}\right) d r_{1} d r_{2} d V_{2} d V_{1} d \omega .
\end{aligned}
$$

We now introduce two collision points $u$ and $u_{*}$ (see figure 5) and replace the collision " $\left(v, v_{*}\right)$ gives $\left(v^{\prime}, v_{*}^{\prime}\right)$ " by the two collisions " $\left(v, u_{*}\right)$ gives $\left(v_{*}^{\prime}, u\right)$ " and " $\left(u, v_{*}\right)$ gives $\left(u_{*}, v^{\prime}\right)$ ".

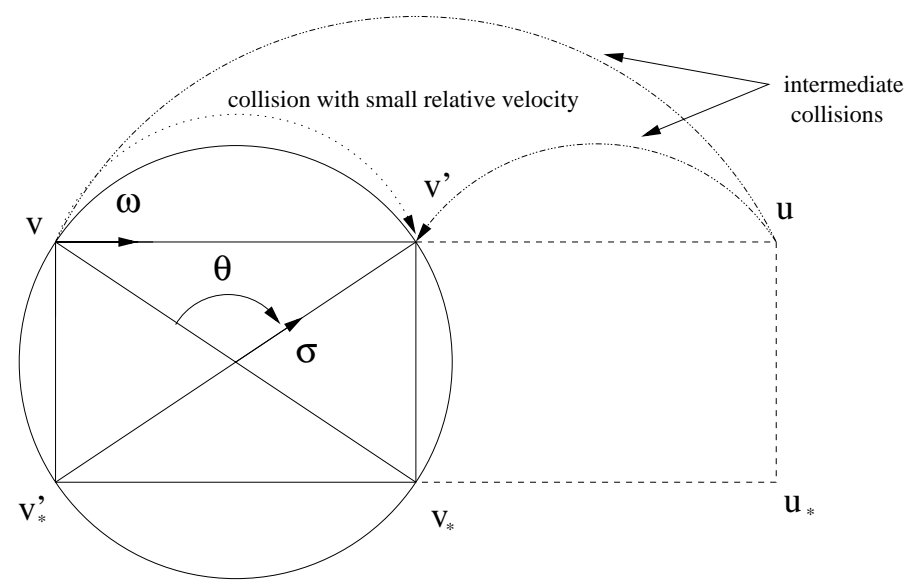

FIGURE 5: Introduction of an intermediate collision. 
Then, we shall use the following "triangular" inequality on the collision points:

$$
\begin{aligned}
{\left[\left(h(v)+h\left(v_{*}\right)\right)-\left(h\left(v^{\prime}\right)+h\left(v_{*}^{\prime}\right)\right)\right]^{2} \leq } & 2\left[\left(h(v)+h\left(u_{*}\right)\right)-\left(h(u)+h\left(v_{*}^{\prime}\right)\right)\right]^{2} \\
& +2\left[\left(h(u)+h\left(v_{*}\right)\right)-\left(h\left(v^{\prime}\right)+h\left(u_{*}\right)\right)\right]^{2} .
\end{aligned}
$$

Recall that $\int_{\mathbb{R}} e^{-r^{2}} d r=\sqrt{\pi}$. Let us add a third artificial integration variable $r_{3}$ on $\mathbb{R}$

$$
\begin{gathered}
D_{1}^{\mathcal{B} o}(h) \geq \frac{1}{4 \sqrt{\pi}} \int_{\mathbb{S}^{N-1}} \int_{V_{1} \in \omega^{\perp}} \int_{V_{2} \in \omega^{\perp}} e^{-\left|V_{1}\right|^{2}-\left|V_{2}\right|^{2}} \int_{r_{1} \in \mathbb{R}} \int_{r_{2} \in \mathbb{R}} \int_{r_{3} \in \mathbb{R}} \Phi\left(\left|r_{2}-r_{1}\right|\right) \tilde{b}\left(\theta_{1,2}\right) \\
k\left(r_{1} \omega+V_{1}, r_{2} \omega+V_{2}, r_{2} \omega+V_{1}, r_{1} \omega+V_{2}\right) e^{-r_{1}^{2}-r_{2}^{2}-r_{3}^{2}} d r_{1} d r_{2} d r_{3} d V_{1} d V_{2} d \omega .
\end{gathered}
$$

From now on, indexes of $\theta$ denote the points which are chosen to compute the angle. Now we rename $r_{1}, r_{2}, r_{3}$ first in $r_{1}, r_{3}, r_{2}$, secondly in $r_{3}, r_{2}, r_{1}$ and we take the mean of these two quantities. We get

$$
\begin{aligned}
D_{1}^{\mathcal{B} o}(h) \geq & \frac{1}{8 \sqrt{\pi}} \int_{\mathbb{S}^{N-1}} \int_{V_{1} \in \omega^{\perp}} \int_{V_{2} \in \omega^{\perp}} e^{-\left|V_{1}\right|^{2}-\left|V_{2}\right|^{2}} \int_{r_{1} \in \mathbb{R}} \int_{r_{2} \in \mathbb{R}} \int_{r_{3} \in \mathbb{R}} e^{-r_{1}^{2}-r_{2}^{2}-r_{3}^{2}} \\
& {\left[\tilde{b}\left(\theta_{1,3}\right) \Phi\left(\left|r_{3}-r_{1}\right|\right) k\left(r_{1} \omega+V_{1}, r_{3} \omega+V_{2}, r_{3} \omega+V_{1}, r_{1} \omega+V_{2}\right)\right.} \\
& \left.+\tilde{b}\left(\theta_{2,3}\right) \Phi\left(\left|r_{2}-r_{3}\right|\right) k\left(r_{3} \omega+V_{1}, r_{2} \omega+V_{2}, r_{2} \omega+V_{1}, r_{3} \omega+V_{2}\right)\right] \\
& d r_{1} d r_{2} d r_{3} d V_{1} d V_{2} d \omega .
\end{aligned}
$$

Then,

$$
\begin{aligned}
D_{1}^{\mathcal{B} o}(h) \geq & \frac{1}{8 \sqrt{\pi}} \int_{\mathbb{S}^{N-1}} \int_{V_{1} \in \omega^{\perp}} \int_{V_{2} \in \omega^{\perp}} e^{-\left|V_{1}\right|^{2}-\left|V_{2}\right|^{2}} \int_{r_{1} \in \mathbb{R}} \int_{r_{2} \in \mathbb{R}} \int_{r_{3} \in \mathbb{R}} \\
& \min \left\{\tilde{b}\left(\theta_{1,3}\right) \Phi\left(\left|r_{3}-r_{1}\right|\right), \tilde{b}\left(\theta_{2,3}\right) \Phi\left(\left|r_{2}-r_{3}\right|\right)\right\} \\
& {\left[k\left(r_{1} \omega+V_{1}, r_{3} \omega+V_{2}, r_{3} \omega+V_{1}, r_{1} \omega+V_{2}\right)+\right.} \\
& \left.k\left(r_{3} \omega+V_{1}, r_{2} \omega+V_{2}, r_{2} \omega+V_{1}, r_{3} \omega+V_{2}\right)\right] \\
& e^{-r_{1}^{2}-r_{2}^{2}-r_{3}^{2}} d r_{1} d r_{2} d r_{3} d V_{1} d V_{2} d \omega .
\end{aligned}
$$

Now we use the following triangular inequality above-mentioned which means translated on $k$

$$
\begin{aligned}
k\left(r_{1} \omega+V_{1}, r_{2} \omega+V_{2}, r_{2} \omega\right. & \left.+V_{1}, r_{1} \omega+V_{2}\right) \\
\leq & 2 k\left(r_{1} \omega+V_{1}, r_{3} \omega+V_{2}, r_{3} \omega+V_{1}, r_{1} \omega+V_{2}\right) \\
& +2 k\left(r_{3} \omega+V_{1}, r_{2} \omega+V_{2}, r_{2} \omega+V_{1}, r_{3} \omega+V_{2}\right) .
\end{aligned}
$$


Plugging it in (2.4), we get

$$
\begin{aligned}
D_{1}^{\mathcal{B} o}(h) \geq \frac{1}{16 \sqrt{\pi}} \int_{\mathbb{S}^{N-1}} \int_{V_{1} \in \omega^{\perp}} \int_{V_{2} \in \omega^{\perp}} e^{-\left|V_{1}\right|^{2}-\left|V_{2}\right|^{2}} \int_{r_{1} \in \mathbb{R}} \int_{r_{2} \in \mathbb{R}} \int_{r_{3} \in \mathbb{R}} \\
\quad \min \left\{\tilde{b}\left(\theta_{1,3}\right) \Phi\left(\left|r_{3}-r_{1}\right|\right), \tilde{b}\left(\theta_{2,3}\right) \Phi\left(\left|r_{2}-r_{3}\right|\right)\right\} \\
k\left(r_{1} \omega+V_{1}, r_{2} \omega+V_{2}, r_{2} \omega+V_{1}, r_{1} \omega+V_{2}\right) e^{-r_{1}^{2}-r_{2}^{2}-r_{3}^{2}} d r_{1} d r_{2} d r_{3} d V_{1} d V_{2} d \omega,
\end{aligned}
$$

which yields

$$
\begin{aligned}
D_{1}^{\mathcal{B} o}(h) \geq & \frac{1}{16 \sqrt{\pi}} \int_{\mathbb{S}^{N-1}} \int_{V_{1} \in \omega^{\perp}} \int_{V_{2} \in \omega^{\perp}} e^{-\left|V_{1}\right|^{2}-\left|V_{2}\right|^{2}} \int_{r_{1} \in \mathbb{R}} \int_{r_{2} \in \mathbb{R}} \\
& \left(\int_{r_{3} \in \mathbb{R}} \min \left\{\tilde{b}\left(\theta_{1,3}\right) \Phi\left(\left|r_{3}-r_{1}\right|\right), \tilde{b}\left(\theta_{2,3}\right) \Phi\left(\left|r_{2}-r_{3}\right|\right)\right\} e^{-r_{3}^{2}} d r_{3}\right) \\
& k\left(r_{1} \omega+V_{1}, r_{2} \omega+V_{2}, r_{2} \omega+V_{1}, r_{1} \omega+V_{2}\right) e^{-r_{1}^{2}-r_{2}^{2}} d r_{1} d r_{2} d V_{1} d V_{2} d \omega .
\end{aligned}
$$

We now restrict the domain of integration for $r_{3}$ to the set

$$
\mathcal{D}_{r_{1}, r_{2}}=\left\{r_{3} \in \mathbb{R}|| r_{3}-r_{1}|\geq| r_{1}-r_{2} \mid \text { and }\left|r_{2}-r_{3}\right| \geq\left|r_{1}-r_{2}\right|\right\} .
$$

Since $\tilde{b}$ is non-decreasing, and

$$
\cos \theta=\frac{\left|V_{1}-V_{2}\right|^{2}-\left|r_{1}-r_{2}\right|^{2}}{\left|V_{1}-V_{2}\right|^{2}+\left|r_{1}-r_{2}\right|^{2}}
$$

which is non-increasing with respect to $\left|r_{1}-r_{2}\right|$ when $V_{1}, V_{2}$ are kept frozen, it is easy to check that on this domain we have $\theta_{1,3} \geq \theta_{1,2}$ and $\theta_{2,3} \geq \theta_{1,2}$ and thus $\tilde{b}\left(\theta_{1,3}\right) \geq \tilde{b}\left(\theta_{1,2}\right)$ and $\tilde{b}\left(\theta_{2,3}\right) \geq \tilde{b}\left(\theta_{1,2}\right)$. Therefore we get

$$
\begin{aligned}
D_{1}^{\mathcal{B} o}(h) \geq & \frac{1}{16 \sqrt{\pi}} \int_{\mathbb{S}^{N-1}} \int_{V_{1} \in \omega^{\perp}} \int_{V_{2} \in \omega^{\perp}} e^{-\left|V_{1}\right|^{2}-\left|V_{2}\right|^{2}} \int_{r_{1} \in \mathbb{R}} \int_{r_{2} \in \mathbb{R}} \\
& \left(\int_{r_{3} \in \mathcal{D}_{r_{1}, r_{2}}} \min \left\{\Phi\left(\left|r_{3}-r_{1}\right|\right), \Phi\left(\left|r_{2}-r_{3}\right|\right)\right\} e^{-r_{3}^{2}} d r_{3}\right) \tilde{b}\left(\theta_{1,2}\right) \\
& k\left(r_{1} \omega+V_{1}, r_{2} \omega+V_{2}, r_{2} \omega+V_{1}, r_{1} \omega+V_{2}\right) e^{-r_{1}^{2}-r_{2}^{2}} d r_{1} d r_{2} d V_{1} d V_{2} d \omega .
\end{aligned}
$$

Under assumption (1.2), an easy computation leads to

$$
\left(\int_{r_{3} \in \mathcal{D}_{r_{1}, r_{2}}} \min \left\{\Phi\left(\left|r_{3}-r_{1}\right|\right), \Phi\left(\left|r_{2}-r_{3}\right|\right)\right\} e^{-\left|r_{3}\right|^{2}} d r_{3}\right) \geq c_{\Phi} \sqrt{\pi} e^{-4 R^{2}}>0
$$

as soon as $\left|r_{1}-r_{2}\right| \leq R$, i.e.,

$$
\left(\int_{r_{3} \in \mathcal{D}_{r_{1}, r_{2}}} \min \left\{\Phi\left(\left|r_{3}-r_{1}\right|\right), \Phi\left(\left|r_{2}-r_{3}\right|\right)\right\} e^{-\left|r_{3}\right|^{2}} d r_{3}\right) \geq c_{\Phi} \sqrt{\pi} e^{-4 R^{2}} 1_{\left|r_{1}-r_{2}\right| \leq R}
$$


By taking the mean of this estimate and the one obtained by replacing $\Phi$ by its bound from below $c_{\Phi} 1_{r \geq R}$, we deduce that

$$
\begin{gathered}
D_{1}^{\mathcal{B} o}(h) \geq \min \left(\frac{c_{\Phi} e^{-4 R^{2}}}{8}, \frac{c_{\Phi}}{2}\right) \frac{1}{4} \int_{\mathbb{S}^{N-1}} \int_{V_{1} \in \omega^{\perp}} \int_{V_{2} \in \omega^{\perp}} e^{-\left|V_{1}\right|^{2}-\left|V_{2}\right|^{2}} \int_{r_{1} \in \mathbb{R}} \int_{r_{2} \in \mathbb{R}} \\
k\left(r_{1} \omega+V_{1}, r_{2} \omega+V_{2}, r_{2} \omega+V_{1}, r_{1} \omega+V_{2}\right) \tilde{b}(\theta) e^{-r_{1}^{2}-r_{2}^{2}} d r_{1} d r_{2} d V_{1} d V_{2} d \omega .
\end{gathered}
$$

If we now go back to the classical representation and simplify the minimum, we obtain

$$
\begin{aligned}
D_{1}^{\mathcal{B} o}(h) & \geq\left(\frac{c_{\Phi} e^{-4 R^{2}}}{8}\right) \frac{1}{4} \int_{\mathbb{S}^{N-1}} \int_{\mathbb{R}^{N}} \int_{\mathbb{R}^{N}} M M_{*} k\left(v, v_{*}, v^{\prime}, v_{*}^{\prime}\right) d \sigma d v d v_{*} \\
& =\left(\frac{c_{\Phi} e^{-4 R^{2}}}{8}\right) D_{0}^{\mathcal{B} o}(h)
\end{aligned}
$$

which concludes the proof of the lemma.

The proof of Theorem 1.1 is a straightforward consequence of inequalities (2.1) and (2.3).

\section{The linearized Landau operator}

We now prove Theorem 1.2. The idea here is to take the grazing collisions limit in some inequalities on the linearized Boltzmann operator obtained thanks to the geometrical method used in Section 2. In fact, the most natural idea would have been to look for a geometrical property on the linearized Landau operator similar to the triangular inequality used for the linearized Boltzmann operator. But as collision circles reduce to lines in the grazing limit, the triangular inequality becomes trivial, and thus does not seem sufficient to apply the method of section 2. It could be linked to the fact that in the grazing collisions limit one loses some information on the geometry of the collision.

The problem that has to be tackled is to keep track of the angular collision kernel $b$. In fact we need it only for particular $b$, namely

$$
b_{\varepsilon}(\theta)=\frac{j_{\varepsilon}(\theta)}{\varepsilon^{2} \sin ^{N-2} \frac{\theta}{2}}
$$

where $j_{\varepsilon}(\theta)=j(\theta / \varepsilon) / \varepsilon$ is a sequence of mollifiers (approximating $\delta_{\theta=0}$ ) with compact support in $[0, \pi / 2]$ and non-increasing on this interval. It is easy to see that $\widetilde{b}_{\varepsilon}=2^{N-1} \sin ^{N-2}\left(\frac{\theta}{2}\right) b_{\varepsilon}$ is also non-increasing on $[0, \pi]$. Following the same strategy as in Lemma 2.2 but keeping track of the angular part of the collision kernel, one obtains 
Lemma 3.1. Under the assumptions (1.1), (1.2), (1.3), plus the assumption that $\tilde{b}=2^{N-1} \sin ^{N-2}\left(\frac{\theta}{2}\right) b$ is non-increasing, one gets for all $h \in L^{2}(M)$

$$
D_{b, \Phi}^{\mathcal{B} o}(h) \geq\left(\frac{c_{\Phi} \beta_{R}}{8 \alpha_{N}}\right) D_{b, 1}^{\mathcal{B} o}(h)
$$

with

$$
\alpha_{N}=\int_{\mathbb{R}^{N-1}} e^{-|V|^{2}} d V, \quad \beta_{R}=\int_{\left\{V \in \mathbb{R}^{N-1}|| V \mid \geq 2 R\right\}} e^{-|V|^{2}} d V
$$

Here $D_{b, \Phi}^{\mathcal{B} o}$ stands for the entropy production functional with $B=\Phi b$ and $D_{b, 1}^{\mathcal{B} o}$ stands for the entropy production functional with $B=b$.

Proof. The geometrical idea of Lemma 2.2 can be applied to the variables $V_{1}, V_{2}$. Let us thus introduce two collisions points $u$ and $u_{*}$ (see figure 6) and replace the collision " $\left(v, v_{*}\right)$ gives $\left(v^{\prime}, v_{*}^{\prime}\right)$ " by the two collisions " $\left(v, u_{*}\right)$ gives $\left(v^{\prime}, u\right)$ " and " $\left(u, v_{*}\right)$ gives $\left(u_{*}, v_{*}^{\prime}\right)$ ".

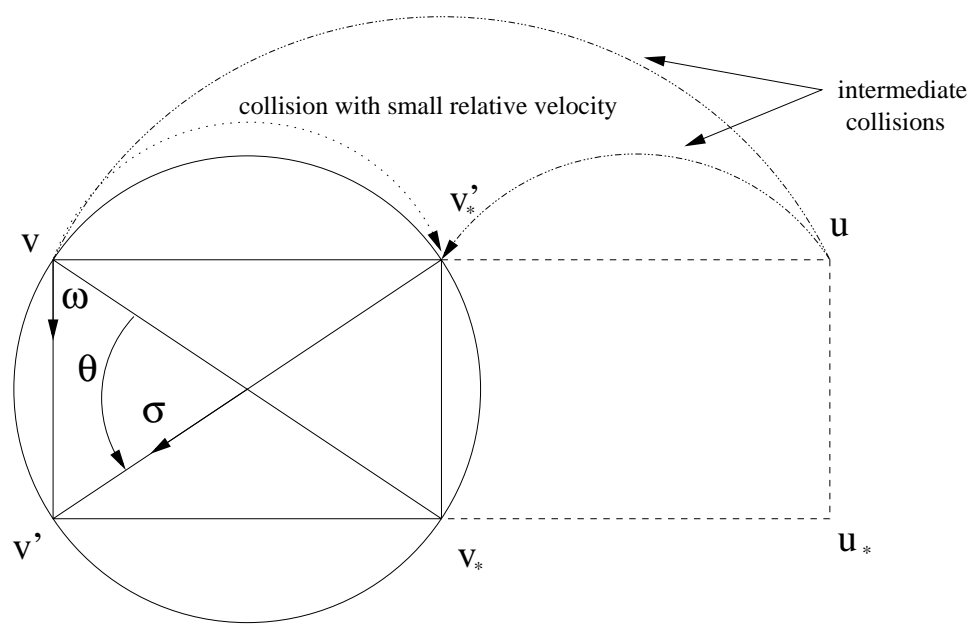

FIGURE 6: Introduction of an intermediate collision.

Then, we shall use the following "triangular" inequality on the collision points:

$$
\begin{aligned}
{\left[\left(h(v)+h\left(v_{*}\right)\right)-\left(h\left(v^{\prime}\right)+h\left(v_{*}^{\prime}\right)\right)\right]^{2} \leq } & 2\left[\left(h(v)+h\left(u_{*}\right)\right)-\left(h\left(v^{\prime}\right)+h(u)\right)\right]^{2} \\
& +2\left[\left(h(u)+h\left(v_{*}\right)\right)-\left(h\left(u_{*}\right)+h\left(v_{*}^{\prime}\right)\right)\right]^{2} .
\end{aligned}
$$

Now we introduce an artificial third variable $V_{3}$ on $\omega^{\perp}$. Let us denote

$$
\alpha_{N}=\int_{\mathbb{R}^{N-1}} e^{-|V|^{2}} d V
$$


By inverting either $V_{1}$ and $V_{3}$ or $V_{2}$ and $V_{3}$, taking the mean, and using the "triangular" inequality above-mentioned we get

$$
\begin{aligned}
D_{b, \Phi}^{\mathcal{B} o}(h) \geq \frac{1}{16 \alpha_{N}} & \int_{\mathbb{S}^{N-1}} \int_{r_{1} \in \mathbb{R}} \int_{r_{2} \in \mathbb{R}} e^{-r_{1}^{2}-r_{2}^{2}} \int_{V_{1} \in \omega^{\perp}} \int_{V_{2} \in \omega^{\perp}} \\
& \left(\int_{V_{3} \in \omega^{\perp}} \min \left\{\tilde{b}\left(\theta_{1,3}\right) \Phi\left(\left|V_{3}-V_{1}\right|\right), \tilde{b}\left(\theta_{2,3}\right) \Phi\left(\left|V_{2}-V_{3}\right|\right)\right\} e^{-\left|V_{3}\right|^{2}} d V_{3}\right) \\
& k\left(r_{1} \omega+V_{1}, r_{2} \omega+V_{2}, r_{2} \omega+V_{1}, r_{1} \omega+V_{2}\right) e^{-\left|V_{1}\right|^{2}-\left|V_{2}\right|^{2}} d r_{1} d r_{2} d V_{1} d V_{2} d \omega .
\end{aligned}
$$

Let us now restrict the integration along $V_{3}$ to the domain

$$
\mathcal{D}_{V_{1}, V_{2}}=\left\{V_{3}|| V_{3}-V_{1}|\geq| V_{1}-V_{2} \mid \text { and }\left|V_{2}-V_{3}\right| \geq\left|V_{1}-V_{2}\right|\right\} \text {. }
$$

Then since the expression

$$
\cos \theta=\frac{\left|V_{1}-V_{2}\right|^{2}-\left|r_{1}-r_{2}\right|^{2}}{\left|V_{1}-V_{2}\right|^{2}+\left|r_{1}-r_{2}\right|^{2}}
$$

is non-decreasing according to $\left|V_{1}-V_{2}\right|$ when $r_{1}, r_{2}$ are kept frozen, and $\tilde{b}$ is non-increasing, we get $\theta_{1,3} \leq \theta_{1,2}$ and $\theta_{2,3} \leq \theta_{1,2}$ (see figure 6) and so $\tilde{b}\left(\theta_{1,3}\right) \geq \tilde{b}\left(\theta_{1,2}\right)$ and $\tilde{b}\left(\theta_{2,3}\right) \geq \tilde{b}\left(\theta_{1,2}\right)$. Consequently

$$
\begin{aligned}
\left.D_{b, \Phi}^{\mathcal{B o o}}(h) \geq \frac{1}{16 \alpha_{N}} \int_{\mathbb{S}^{N-1}} \int_{r_{1} \in \mathbb{R}} \int_{r_{2} \in \mathbb{R}} d r_{2} e^{-r_{1}^{2}-r_{2}^{2}} \int_{V_{1} \in \omega^{\perp}} \int_{V_{2} \in \omega^{\perp}} \min \left\{\Phi\left(\left|V_{3}-V_{1}\right|\right), \Phi\left(\left|V_{2}-V_{3}\right|\right)\right\} e^{-\left|V_{3}\right|^{2}} d V_{3}\right) \tilde{b}\left(\theta_{1,2}\right) \\
\left(\int_{V_{3} \in \mathcal{D}_{V_{1}, V_{2}}} \min e^{-\left|V_{1}\right|^{2}-\left|V_{2}\right|^{2}} d r_{1} d r_{2} d V_{1} d V_{2} d \omega .\right.
\end{aligned}
$$

Under assumption (1.2), an easy computation leads to

$$
\begin{aligned}
\left(\int_{V_{3} \in \mathcal{D}_{V_{1}, V_{2}}} \min \left\{\Phi\left(\left|V_{3}-V_{1}\right|\right), \Phi\left(\left|V_{2}-V_{3}\right|\right)\right\} e^{-\left|V_{3}\right|^{2}} d V_{3}\right) & \\
& \geq c_{\Phi} \int_{\left\{V \in \mathbb{R}^{N-1}|| V \mid \geq 2 R\right\}} e^{-|V|^{2}} d V=c_{\Phi} \beta_{R}>0
\end{aligned}
$$

as soon as $\left|V_{1}-V_{2}\right| \leq R$, i.e.,

$$
\left(\int_{V_{3} \in \mathcal{D}_{V_{1}, V_{2}}} \min \left\{\Phi\left(\left|V_{3}-V_{1}\right|\right), \Phi\left(\left|V_{2}-V_{3}\right|\right)\right\} e^{-\left|V_{3}\right|^{2}} d V_{3}\right) \geq c_{\Phi} \beta_{R} 1_{\left|V_{1}-V_{2}\right| \leq R} .
$$


Taking the mean of this estimate and the one obtained by the trivial lower bound $\Phi(r) \geq c_{\Phi} 1_{\{r \geq R\}}$, we get in the end

$D_{b, \Phi}^{\mathcal{B} o}(h) \geq \min \left(\frac{c_{\Phi} \beta_{R}}{8 \alpha_{N}}, \frac{c_{\Phi}}{2}\right) \frac{1}{4} \int_{\mathbb{S}^{N-1}} \int_{\mathbb{R}^{N}} \int_{\mathbb{R}^{N}} b(\theta) M M_{*}\left[h_{*}^{\prime}+h^{\prime}-h-h_{*}\right]^{2} d \sigma d v_{*} d v$,

which yields

$$
D_{b, \Phi}^{\mathcal{B}_{0}}(h) \geq\left(\frac{c_{\Phi} \beta_{R}}{8 \alpha_{N}}\right) D_{b, 1}^{\mathcal{B o}_{0}}(h)
$$

and concludes the proof of the lemma.

We now have to take the grazing collisions limit in the entropy production functional to prove inequality (1.6) of Theorem 1.2 (this limit is essentially well-known, see for instance [9]).

Lemma 3.2. Let us consider $b_{\varepsilon}$ as defined in (3.1) and $\Phi$ satisfying assumption (1.2). Then for a given $h \in L^{2}(M)$,

$$
D_{b_{\varepsilon}, \Phi}^{\mathcal{B} o}(h) \underset{\varepsilon \rightarrow 0}{\longrightarrow} c_{N, j} D_{\Phi}^{\mathcal{L} a}(h)
$$

where

$$
c_{N, j}=\frac{2^{N-5}\left|\mathbb{S}^{N-2}\right|}{N-1}\left(\int_{o}^{\pi} j(\chi) \chi^{2} d \chi\right)
$$

depends only on the dimension $N$ and the mollifier $j . D_{b_{\varepsilon}, \Phi}^{\mathcal{B o}}$ stands for the Boltzmann entropy production functional with $B=\Phi b_{\varepsilon}$, and $D_{\Phi}^{\mathcal{L} a}$ stands for the Landau entropy production functional with collision kernel $\Phi$.

Proof. The idea of the proof is to expand the expression for small $\varepsilon$ and is very similar to what is done in [9]. Let us write the angular vector $\sigma$

$$
\sigma=\frac{v-v_{*}}{\left|v-v_{*}\right|} \cos (\theta)+\mathbf{n} \sin (\theta)
$$

where $\mathbf{n}$ is a unit vector in $\left(v-v_{*}\right)^{\perp}$. Therefore, we shall write

$$
\begin{aligned}
D_{b_{\varepsilon}, \Phi}^{\mathcal{B}_{0}}(h)=\frac{1}{4} \int_{\mathbb{R}^{N}} \int_{\mathbb{R}^{N}} \Phi\left(\left|v-v_{*}\right|\right) M M_{*} \int_{\mathbb{S}^{N-2}\left(\left(v-v_{*}\right)^{\perp}\right)} \int_{\theta=0}^{\pi} b_{\varepsilon}(\theta) \\
\quad\left[h\left(v-\frac{v-v_{*}}{2}(1-\cos (\theta))+\frac{\left|v-v_{*}\right|}{2} \mathbf{n} \sin (\theta)\right)\right. \\
\left.\quad+h\left(v_{*}+\frac{v-v_{*}}{2}(1-\cos (\theta))-\frac{\left|v-v_{*}\right|}{2} \mathbf{n} \sin (\theta)\right)-h(v)-h\left(v_{*}\right)\right]^{2} \\
\quad \sin ^{N-2} \theta d \theta d \mathbf{n} d v d v_{*},
\end{aligned}
$$

where $\mathbb{S}^{N-2}\left(\left(v-v_{*}\right)^{\perp}\right)$ denotes the unit sphere in $\left(v-v_{*}\right)^{\perp}$. 
Let us now focus on the integral on $\theta$

$$
\begin{aligned}
& \int_{\theta=0}^{\pi} b_{\varepsilon}(\theta)\left[h\left(v-\frac{v-v_{*}}{2}(1-\cos (\theta))+\frac{\left|v-v_{*}\right|}{2} \mathbf{n} \sin (\theta)\right)\right. \\
& \left.\quad+h\left(v_{*}+\frac{v-v_{*}}{2}(1-\cos (\theta))-\frac{\left|v-v_{*}\right|}{2} \mathbf{n} \sin (\theta)\right)-h(v)-h\left(v_{*}\right)\right]^{2} \sin ^{N-2} \theta d \theta,
\end{aligned}
$$

and make the change of variables $\chi=\theta / \varepsilon$. We get

$$
\begin{gathered}
\int_{\chi=0}^{\pi} \frac{\sin ^{N-2}(\varepsilon \chi)}{\sin ^{N-2}\left(\frac{\varepsilon \chi}{2}\right)} \frac{j(\chi)}{\varepsilon^{2}}\left[h\left(v-\frac{v-v_{*}}{2}(1-\cos (\varepsilon \chi))+\frac{\left|v-v_{*}\right|}{2} \mathbf{n} \sin (\varepsilon \chi)\right)\right. \\
\left.+h\left(v_{*}+\frac{v-v_{*}}{2}(1-\cos (\varepsilon \chi))-\frac{\left|v-v_{*}\right|}{2} \mathbf{n} \sin (\varepsilon \chi)\right)-h(v)-h\left(v_{*}\right)\right]^{2} d \chi
\end{gathered}
$$

i.e., for small $\varepsilon$,

$$
\int_{\chi=0}^{\pi}\left(2^{N-2}+O(\varepsilon)\right) \frac{j(\chi)}{\varepsilon^{2}}\left(\frac{\left|v-v_{*}\right|}{2}\right)^{2}\left[\varepsilon \chi \mathbf{n} \cdot\left(\nabla_{v} h(v)-\nabla_{v_{*}} h\left(v_{*}\right)\right)+O\left(\varepsilon^{2} \chi^{2}\right)\right]^{2} d \chi,
$$

which writes

$$
\begin{aligned}
& \left|v-v_{*}\right|^{2} \int_{\chi=0}^{\pi} 2^{N-4} j(\chi) \chi^{2}\left[\mathbf{n} \cdot\left(\nabla_{v} h(v)-\nabla_{v_{*}} h\left(v_{*}\right)\right)\right]^{2} d \chi+O(\varepsilon) \\
& =2^{N-4}\left(\int_{o}^{\pi} j(\chi) \chi^{2} d \chi\right)\left|v-v_{*}\right|^{2}\left[\mathbf{n} \cdot\left(\nabla_{v} h(v)-\nabla_{v_{*}} h\left(v_{*}\right)\right)\right]^{2}+O(\varepsilon) .
\end{aligned}
$$

As the unit vector $\mathbf{n}$ is orthogonal to $\frac{v-v_{*}}{\left|v-v_{*}\right|}$, we can introduce here the orthogonal projection onto $\left(v-v_{*}\right)^{\perp}$

$$
\begin{aligned}
D_{b_{\varepsilon}, \Phi}^{\mathcal{B} o}(h)= & \frac{2^{N-4}}{4}\left(\int_{o}^{\pi} j(\chi) \chi^{2} d \chi\right) \int_{\mathbb{R}^{N}} \int_{\mathbb{R}^{N}} \int_{\mathbb{S}^{N-2}\left(\left(v-v_{*}\right)^{\perp}\right)} \Phi\left(\left|v-v_{*}\right|\right)\left|v-v_{*}\right|^{2} \\
& {\left[\mathbf{n} \cdot \Pi_{\left(v-v_{*}\right)^{\perp}}\left(\nabla_{v} h(v)-\nabla_{v_{*}} h\left(v_{*}\right)\right)\right]^{2} M M_{*} d \mathbf{n} d v d v_{*}+O(\varepsilon) . }
\end{aligned}
$$

It is straightforward to see that

$$
\int_{\mathbb{S}^{N-2}\left(\left(v-v_{*}\right)^{\perp}\right)}(\mathbf{n} \cdot u)^{2} d \mathbf{n}=\zeta_{N}\|u\|^{2}
$$

with, for any $\mathbf{u} \in \mathbb{S}^{N-2}$

$$
\zeta_{N}=\int_{\mathbb{S}^{N-2}}(\mathbf{u} \cdot \mathbf{n})^{2} d \mathbf{n}=\frac{\left|\mathbb{S}^{N-2}\right|}{N-1} .
$$


Thus we get in the end

$$
\begin{aligned}
D_{b_{\varepsilon}, \Phi}^{\mathcal{B} o}(h) & =\frac{\left|\mathbb{S}^{N-2}\right| 2^{N-4}}{4(N-1)}\left(\int_{o}^{\pi} j(\chi) \chi^{2} d \chi\right) \\
& \int_{\mathbb{R}^{N}} \int_{\mathbb{R}^{N}}\left|v-v_{*}\right|^{2} \Phi\left(\left|v-v_{*}\right|\right) M M_{*}|| \nabla_{v} h(v)-\left.\nabla_{v_{*}} h\left(v_{*}\right)\right|^{2} d v_{*} d v+O(\varepsilon) \\
& =\frac{\left|\mathbb{S}^{N-2}\right| 2^{N-5}}{N-1}\left(\int_{o}^{\pi} j(\chi) \chi^{2} d \chi\right) D_{\Phi}^{\mathcal{L} a}(h)+O(\varepsilon) .
\end{aligned}
$$

This concludes the proof of Lemma 3.2.

Coming back to the proof of Theorem 1.2, we first prove (1.6): we write down inequality (3.2) on $D_{\Phi, b_{\varepsilon}}^{\mathcal{B} o}$ since $\tilde{b}_{\varepsilon}$ is non-increasing, and we apply Lemma 3.2 on each term, which gives

$$
D^{\mathcal{L} a}(h) \geq C_{\Phi}^{\mathcal{L} a} D_{0}^{\mathcal{L} a}(h),
$$

where

$$
C_{\Phi}^{\mathcal{L} a}=\left(\frac{c_{\Phi} \beta_{R}}{8 \alpha_{N}}\right)
$$

Inequality (1.7) follows immediately.

It remains to prove the lower bound (1.8) on the first non-zero eigenvalue of the linearized Landau operator for Maxwellian molecules in dimension 3. Let us denote by $\lambda_{0, b_{\varepsilon}}^{\mathcal{B}_{\mathrm{s}}}$ the first non-zero eigenvalue for the Boltzmann linearized operator with $B=b_{\varepsilon}$ : for all $h \in L^{2}(M)$ orthogonal in $L^{2}(M)$ to $1, v,|v|^{2}$,

$$
D_{b_{\varepsilon}}^{\mathcal{B} o}(h) \geq\left|\lambda_{0, b_{\varepsilon}}^{\mathcal{B} o}\right|\|h\|_{L^{2}(M)}^{2} .
$$

We apply Lemma 3.2 to this inequality which leads to

$$
D_{0}^{\mathcal{L} a}(h) \geq \frac{\lim _{\varepsilon \rightarrow 0}\left|\lambda_{0, b_{\varepsilon}}^{\mathcal{B} o}\right|}{c_{3, j}}\|h\|_{L^{2}(M)}^{2}
$$

for all $h \in L^{2}(M)$ orthogonal in $L^{2}(M)$ to $1, v,|v|^{2}$. An explicit formula for $\left|\lambda_{0, b_{\varepsilon}}^{\mathcal{B}_{0}}\right|$ is given in [1]

$$
\left|\lambda_{0, b_{\varepsilon}}^{\mathcal{B} o}\right|=\pi^{5 / 2} \int_{0}^{\pi} \sin ^{3}(\theta) b_{\varepsilon}(\theta) d \theta
$$

and thus

$$
\lim _{\varepsilon \rightarrow 0}\left|\lambda_{0, b_{\varepsilon}}^{\mathcal{B}_{0}}\right|=2 \pi^{5 / 2}\left(\int_{0}^{\pi} j(\chi) \chi^{2} d \chi\right)
$$

which concludes the proof. 


\section{References}

[1] Bobylëv, A. V.: The theory of the nonlinear spatially uniform Boltzmann equation for Maxwell molecules, In Mathematical physics reviews, Vol. 7, 111-233. Soviet Sci. Rev. Sect. C Math. Phys. Rev. 7, Harwood Academic Publ., Chur, 1988.

[2] Caflisch, R. E.: The Boltzmann equation with a soft potential. II. Nonlinear, spatially-periodic. Comm. Math. Phys. 74 (1980), no. 2, 97-109.

[3] Carrilo, J., McCann, R. and Villani, C.: Kinetic equilibration rates for granular media and related equations: entropy dissipation and mass transportation estimates. Rev. Mat. Iberoamericana 19 (2003), no. 3, 971-1018.

[4] Cercignani, C.: The Boltzmann equation and its applications. Applied Mathematical Sciences 67. Springer-Verlag, New York, 1988.

[5] Cercignani, C., Illner, R. And Pulvirenti, M.: The Mathematical theory of dilute gases. Applied Mathematical Sciences 106. Springer-Verlag, New York, 1994.

[6] Czechowski, Z. and Palczewski, A.: Spectrum of the Boltzmann collision operator for radial cut-off potentials. Bull. Acad. Polon. Sci. Sér. Sci. Tech. 28 (1980), no. 9-10, 387-396.

[7] Degond, P. And Lemou, M.: Dispersion relations for the linearized Fokker-Planck equation. Arch. Rational Mech. Anal. 138 (1997), no. 2, 137-167.

[8] Desvillettes, L.: Entropy dissipation rate and convergence in kinetic equations. Comm. Math. Phys. 123 (1989), no. 4, 687-702.

[9] Desvillettes, L.: On asymptotics of the Boltzmann equation when the collisions become grazing. Transport Theory Statist. Phys. 21 (1992), no. 3, 259-276.

[10] Desvillettes, L., Convergence towards the thermodynamical equilibrium. In Trends in applications of mathematics to mechanics (Nice, 1998), 115-126. Monogr. Surv. Pure Appl. Math. 106. Chapman \& Hall/CRC, Boca Raton, FL, 2000.

[11] Desvillettes, L. And Villani, C: On the spatially homogeneous Landau equation for hard potentials. II. $H$-theorem and applications. Comm. Partial Differential Equations 25 (2000), no. 1-2, 261-298.

[12] Ellis, R. S. And Pinsky, M. A.: The first and second fluid approximations to the linearized Boltzmann equation. J. Math. Pures Appl. (9) $\mathbf{5 4}$ (1975), 125-156.

[13] Golse, F. and Poupaud, F.: Un résultat de compacité pour l'équation de Boltzmann avec potentiel mou. Application au problème de demi-espace, C. R. Acad. Sci. Paris Sér. I Math. 303 (1986), no. 12, 583-586.

[14] Klaus, M.: Boltzmann collision operator without cut-off. Helv. Phys. Acta 50 (1977), no. 6, 893-903. 
[15] PAO, Y. P.: Boltzmann collision operator with inverse-power intermolecular potentials. I, II. Comm. Pure Appl. Math. 27 (1974), 407-428, 559-581.

[16] Villani, C.: A review of mathematical topics in collisional kinetic theory. In Handbook of mathematical fluid dynamics, Vol. I, 71-305. NorthHolland, Amsterdam, 2002.

[17] Villani, C.: On the spatially homogeneous Landau equation for Maxwellian molecules. Math. Models Methods Appl. Sci. 8 (1998), no. 6, 957-983.

[18] Villani, C.:, Contribution à l'étude mathématique des collisions en théorie cinétique. Master's thesis, Univ. Paris-Dauphine France, 2000.

[19] Wang Chang, C. S., Uhlenbeck, G. E. and De Boer, J.: Studies in Statistical Mechanics, Vol. V. North-Holland, Amsterdam, 1970.

[20] Wennberg, B.: On an entropy dissipation inequality for the Boltzmann equation. C. R. Acad. Sci. Paris Sér. I Math. 315 (1992), no. 13, 1441-1446.

Recibido: 28 de marzo de 2003

Revisado: 7 de julio de 2003

Céline Baranger

CMLA, ENS Cachan

61 avenue du Président Wilson

94235 Cachan Cedex, FRANCE

Celine.Baranger@cmla.ens-cachan.fr

Clément Mouhot

UMPA, ENS Lyon

46 allée d'Italie

69364 Lyon Cedex 07, FRANCE

cmouhot@umpa.ens-lyon.fr

Support by the European network HYKE, funded by the EC as contract HPRN-CT-200200282, is acknowledged. 